\title{
IL DUELLO NEL RINASCIMENTO E IL RIPENSAMENTO ETICO SUL DUELLO DI FRANCESCO PATRIZ[ ${ }^{[1]}$
}

\author{
Fulvio Šuran \\ Dipartimento di studi in lingua italiana \\ Università Juraj Dobrila di Pola
}

\section{Riassunto}

In questa prima parte del saggio ci soffermeremo in modo particolare al periodo che intercorre tra la seconda metà del XV secolo e la prima metà del XVI secolo, in quanto è in questo lasso di tempo che si trovano serie e chiare testimonianze riguardanti la questione del duello. E questo sia nelle opere letterarie che in quelle riguardanti la precettistica. Per quanto riguarda quelle di precettistica, oltre al gran numero di trattati, è da tener presente anche il gran numero delle loro riedizioni, specialmente di quelli più famosi. Dove, oltre all'immancabile comunanza d'interessi fra poeti epici e trattatisti del duello, si noterà anche una sostanziale identità terminologica - per esempio sia $\mathrm{i}$ poeti sia i trattatisti ritengono che il duello sia una 'prova' di giudizio finale. Questo non comporta, nel contempo, una convergenza di convinzioni filosofiche, in quanto, come si vedrà, il duello letterario differisce da quello reale proprio per la sua diversa comprensione filosofica del combattere. Pertanto con la presente relazione si cercherà di dispiegare la problematica che questo istituto di per sé ha comportato nel periodo storico preso in considerazione.

Il duello infatti si configurava come una questione sociale tutt'altro che irrilevante per la sua connotazione ambivalente di ordalia - quale forma particolare di rapporto fra uomo e sacro - e di inculpata tutela - quale forma particolare di ricorso alla violenza privata a tutela di un fondamentale diritto naturale. In tutti e due i casi si tratta di una forma di onore, sia individuale sia nobiliare, del quale il Patrizi ne discuterà la fondatezza interpretandolo specialmente in qualità di virtù. In questa definizione dell'onore - che il Patrizi affronta con un gusto platonico - si possono scorgere questioni di alta portata politica, che ponevano sotto varia forma la questione dei rapporti tra individuo e Stato. In tal modo il duello d'onore assumeva un aspetto decisivo per quanto riguardava le sue connotazioni sociali e politiche inserendosi nel più ampio dibattito comprendente il problema dell'uso della violenza e dei confini fra violenza privata e violenza pubblica, fra faida e bellum iustum.

Parole chiave: trattatistica del duello, la causa 'giusta', il duello come 'prova', Platone, Iliade, virtù, etica

\footnotetext{
[1] Questo lavoro nasce da un'attenta lettura del Dialogo dell'honore, il Berignano di Francesco Patrizi da Cherso d'Istria (Cres) in Croazia, ed è divisa in due parti. La prima, pubblicata in questo numero, tratta la questione del duello, dellonore e della dignità, propria allordine cavalleresco e allaristocrazia europea, ben messa in mostra dalla trattatistica rinascimentale, all'interno della visione culturale propria al Cinquecento italiano e in contrapposizione a quello teutonico. Nella seconda parte si parlerà invece del suo diniego da parte di pensatori del rango del filosofo istriano di Cherso Francesco Patrizi (Frane Petrić).
} 


\section{Il duello nel Rinascimento}

\section{Introduzione}

E quanto mai necessario soffermarci sulla rinascimentale categorizzazione del duello, quale difesa dellonore, in quanto certi autori contemporanei, per lo più dell'Europa settentrionale, del livello di Victor G. Kiernan ${ }^{[2]}$, escludono categoricamente la paternità mediterranea del duello, anche se ammettono che molti caratteri propri al duellare greco-romano confluiranno nel duello della modernità, ritenendo comunque che i duellanti dell'età moderna trovano la loro vera essenza nell'individualismo proprio al cavaliere medievale più che nella milizia greco-romana che nel collettivismo basava il suo senso d'esistenza.

Mi sembra si tratti di una debolezza filosofica, di un non rendersi conto che la civiltà greca classica rappresenti per l'Europa, ed in genere per il mondo occidentale, un esempio d'individualità, in quanto dell'individuo ha fatto il perno delle proprie speculazioni e riflessioni, sfocianti nel rinascimentale "essere o non essere" dello Shakespeare.

Basterà qui soffermarci sul senso profondo della filosofia greca per scorgervi quella particolare attenzione, che in genere contraddistinguerà tutta la filosofia occidentale, rivolta al soggetto umano e al senso della sua vita. Per rendersi conto di ciò basta analizzare le regole comportamentali che l'uomo greco e i pàides applicavano, dipendentemente alle diverse polis a tale comprensione d'individualità. A tale proposito le olimpiadi rappresentavano il massimo elogio dell'individualità propria all'uomo greco, quale legislazione di sé a se stessi e non imposizione esterna-estranea, ma autodisciplinamento.

Sulla base di questo controllo esercitato, all'interno del singolo individuo, dallanima, o meglio dalla parte razionale della stessa, sulle passioni del corpo e sugli istinti che accomunano l'uomo con l'animale, quale continuo pòlemos tra anima e corpo all'interno dello stesso essere umano, si possono comprendere le ragioni di Massimo Cacciari che in Geo-filosofia dell'Europa $a^{[3]}$ al duello dedica un intero capitolo nel quale, trattando della condizione dell'eroe, la equipara a quella di una continua precarietà di chi può commettere una violazione dell'ordine e contemporaneamente trattenersi all'interno del proprio limite. Due dimensioni, quella di hýbris e d'ordine, che necessariamente convivono in lui per cui il mantenerle in costante armonia è la sfida cui leroe si trova costantemente di fronte nel proprio agire.

In questo senso i duelli omerici davanti alle mura di Troia sono il perfetto esempio di tale armonia polemica, che impedisce agli scontri tra i singoli guerrieri di trasformare il loro combattimento in un semplice e rozzo spargimento di sangue.

Molto probabilmente la realtà fu ben diversa da tali elaborazioni poetiche e filosofiche, ma una cosa è fondamentale, cioè il comprendere che il duello non nasce ed essenzialmente non è una pratica di un ceto ormai in declino che cerca di proteggersi, cioè di salvaguardare la propria separatezza dal resto della società e quindi i propri privilegi. Anche se pare ovvio che, come del resto per ogni creazione da parte dell'uomo, il duello

[2] Kiernan, V. G., Il duello. Onore e aristocrazia nella storia europea, Marsilio, Milano, 1999.

[3] Cacciari, M., Geo-filosofia dell'Europa. Capitolo 3. Gli eroi, Adelphi, Milano, 1994, pp. 79-103. 
andò incontro a corruzione, allontanandosi dal modello originario ed esponendosi in tal modo alle critiche di cui fu oggetto.

Non dimenticando, a tale proposito che a questi mutamenti intrinseci si devono altresì aggiungere anche quelli estrinseci, cioè relativi alla trasformazione della società e degli schemi concettuali. A tale riguardo si deve specificare come non sia accettabile un'analisi del duello che si basi su una serie di pregiudizi e stereotipi culturali che ne facciano emergere soltanto i caratteri esteriori ed inessenziali.

Per quel che riguarda la modalità nell'affrontare questa tematica, cè da dire che - di contro a qualsivoglia retroterra e sostrato culturale e ideologico, da accantonare ai fini di un'oggettiva e veridica ricerca - la corretta analisi di un contenuto è quella di dare spazio alloggetto stesso della ricerca. Non si tratta di mera retorica o di un paràdeigma perfetto ma inapplicabile, quanto del fatto che il contenuto deve venire sottoposto ad un'analisi di verifica, cioè ad una distinzione che ne contraddistingua, senza dividerli, i suoi diversi elementi costitutivi, così che essi mostrino la loro natura e la loro provenienza, guidandoci in tal modo verso l'auspicata comprensione dell'oggetto d'indagine, quale fine ultimo di ogni metodo a tutti gli effetti. Distinguo che è altresì valido anche per la questione inerente il duello. Dal quale, come si vedrà, ne emergerà una rete di connessioni inaspettate che ci permetteranno di collegarlo all'anima greca come sua sorgente naturale.

\section{Origine greca del duello occidentale}

Contrariamente alle comuni opinioni, l'attenzione verso l'individuo non appartiene solamente alla modernità, ma è caratteristica essenziale della cultura e del mondo greco classico. In quanto, pensare che in tale contesto a prevalere sia la dimensione collettiva, sarebbe una limitazione che ci priverebbe di una delle più originali e sentite tematiche di riflessione della grecità. Svariati sono pure gli indizi che portano verso questo tipo d'interpretazione.

Come non accorgersi, per esempio, che uno dei termini e dei concetti chiave ellenici, quello di pòlis appunto, significhi sì città, ma nel senso della dimensione collettiva all'interno della quale il singolo soggetto s'inserisce, potendo in tal modo definirsi un cittadino che nell'interrelazione con gli altri membri della comunità acquista la propria identità sotto forma di "zoon politikon". Ma è altresì vero che l'ordine e la vita della polis si fondano sulla soggettività degli individui, e quindi non in quanto atomi del corpo sociale, ma in quanto questi, con il loro comportamento e condotta verso se stessi ed entro i limiti delle proprie famiglie, in definitiva determinano il buon funzionamento dello stesso ordine sociale costituito.

Non ci si trova qui di fronte ad un sistema artificiale nel quale l'individuo si annulla, come nel Leviathan di Thomas Hobbes. Non più quindi alla ricerca del bene comune, come pensavano gli antichi, quanto alla semplice e banale tutela dal male. In tal modo il diritto formale opera una considerevole riduzione della complessità del mondo reale, per cui sarebbe quanto mai un errore di anacronismo applicare le categorie hobbesiane alla politica e alla società greche. 
Le due dimensioni, quella della famiglia e della città, si basano sulla soggettività dell'individuo. Unicamente un uomo padrone di sé e attivo nei propri confronti è in grado di svolgere sia il ruolo di marito e capofamiglia, sia quello di esercitare il potere politico sotto forma di governante della città. Entriamo in tal modo nel vivo della questione riguardante la padronanza di sé e la condizione di attività-passività nei propri confronti. Espressioni che si riferiscono alla costruzione dell'individualità in quanto fanno riferimento al rapporto che il soggetto instaura con se stesso quanto al dominio delle proprie passioni e dei piaceri (gli aphrodisia), il che platonicamente si traduce nel dominio della parte razionale dell'anima su quella passionale e sensibile.

È ovvio che qui ci si riferisce al Fedro di Platone dove si trova uno di quei miti platonici che, per la loro forza esplicativa, fanno parte dell'iconografia tradizionale. Nel caso che qui ci interessa, si tratta del mito dell'Auriga alla guida del cocchio trainato da due cavalle una delle quali, la cavalla bianca, è docile, si lascia dirigere con facilità e non cerca di abbandonare il percorso intrapreso dall'Auriga, dove invece la cavalla nera è selvaggia e non perde occasione per deviare il cocchio verso il basso.

Compito principale del conducente è quello di cercare di armonizzare le due forze contrapposte dalla cui sfida continua in se stesso dipendeva il destino di se stesso. Non ci deve, quindi, apparire affatto contraddittoria l'espressione nella Repubblica di Platone secondo cui "si è più forti o più deboli di se stessi". Dove più forte, e quindi attivo nei confronti di se stesso, è colui che riesce a dominare le passioni, più debole invece è colui che ne diviene schiavo, ovvero colui che non vuole, o è incapace, di impedire la tirannia dei pathèmata su se stesso ${ }^{[4]}$. Due sono rispettivamente i concetti che indicano sia il processo di autodisciplinamento, di autoformazione del sé come soggetto di desiderio, sia l'esercizio necessario per potere dominare se stessi. Si tratta in effetti di enkratèia e di sophrosùne ${ }^{[5]}$.

A distinguere i due concetti è stato Aristotele che nell'Etica Nicomachea mostra come il primo termine sia la condizione del secondo che di quello dipende.

La cultura greca elaborò tutta una serie di pratiche volontarie e ragionate che come scopo avevano non solo la definizione di canoni comportamentali, secondo i quali l'uomo greco non doveva solo atteggiarsi e dare mostra di sé agli altri, ma il rendere l'uomo "demiurgo a se stesso", in quanto capace di fare della propria vita un'opera d'arte.

Proprio per questa ragione tali pratiche e tale uso di passioni e piaceri sono definibili come arti esistenziali e quindi non sotto forma di un rigido codice normativo, quanto di un insieme di richieste che l'individuo fa a se stesso, aiutato in questo dal contesto sociale. In tal senso da sottolineare come lo stesso principio del dominio di se stessi e su se stessi, quale autocrazia, stia alla base sia del rapporto del singolo con se stesso ed i propri piaceri, sia di quello del marito e/o capofamiglia con la legittima consorte e l'òikos; sia infine di quello del politico con la sua pòlis. Perché un individuo, se incapace di essere attivo e di governare se stesso, come può pretendere di amministrare e dirigere tanto la res privata quanto la res pubblica? Si trattava di una questione di onorabilità e di merito. Concetti che erano già ben noti all'individuo greco che ne faceva una questione di vita o di morte.

[4] Qui il termine greco indica proprio il divenire passivo del soggetto stesso.

[5] Termini che compaiono sempre assieme, pur non essendo sinonimi e mantenendosi distinti, in quanto la prima è controllo dinamico e attivo di sé, dominio consistente in tensione e lotta, mentre la seconda è uno stato generale tra eccesso e insensibilità ai piaceri in cui si decide di agire bene nei confronti di dei e uomini, una condizione che gioca sul piano della staticità. 
In tal senso è interessante la pressione sociale esercitata dal contesto sociale sul giovane. Basti pensare a Pausania che nel Simposio platonico racconta che ad Atene "i genitori ti mettono alle calcagne dei loro figlioli un pedagogo, col preciso incarico di tenerli lontani dai loro corteggiatori, quando i compagni e i coetanei fanno quasi succedere uno scandalo se si accorgono di qualcosa del genere, mentre i più anziani lasciano che dicano e non intervengono a queste esagerate reazioni”" ${ }^{[6]}$.

La moderazione delluomo greco nelle diverse situazioni esistenziali, in questo caso specifico sia dell'amante che dell'amato, era veramente una preoccupazione sociale.

Alla luce degli elementi emersi dalla nostra ricerca, può essere giudicato arrendevole e schiavo della società quel giovane che si conforma e segue ciò che essa gli indica? Rispondere in modo affermativo vuol dire rimanere saldamente ancorati allottica del XX secolo e non sforzarsi di comprendere che si tratta di un di per sé diverso punto di vista, giacché esso nasce proprio nel contesto del mondo greco incarnandone i principi di autocontrollo e di estetica del vivere sociale. Basti qui, per iniziare, prendere in esame un passo del poema omerico, l'Iliade seguendo in esso la trattatistica greca che, come esempi di armonia anima-corpo, di chrèsis aphrodisiòn indica sia l'atleta sia il guerriero, in quanto capaci entrambi di frenare gli impulsi fisici, di essere temperanti. In tal senso l'opera di Omero rappresenta un susseguirsi di eroi e di "dei" che, pur dovendo battersi e macchiarsi del sangue dei nemici, non si abbandonano mai alla cieca ed ebbra violenza; dove non cè mai un cedimento all'hýbris, e questo tanto negli scontri di massa, quanto nei singoli duelli.

Il Libro VII dell'Iliade si apre con una scena di combattimento, cosa che di per sé non desta affatto stupore, visto l'argomento trattato, tuttavia si assiste a qualcosa di singolare, in quanto gli dei, Atena e Apollo, decidono di comune accordo che è necessaria una momentanea cessazione degli scontri, una pausa. A tale proposito queste parole il sire figlio di Zeus Apollo rivolse alla dea Atena occhio azzurro:

\footnotetext{
"Ma se tu mascoltassi, questo sarebbe meglio: facciam finire adesso la pugna e la battaglia per oggi; poi pugneremo di nuovo, sinché la fine trovino d'Ilio, già che tanto piaque nel cuore a voi dee, d'abbattere questa rocca" ${ }^{\text {"[7] }}$.
}

Alla domanda della dea Atena di come intendesse egli "por fine alla lotta dei forti?", Apollo, di contro alla lotta degli àndres, propone proprio uno scontro tra due campioni con queste parole:

Destiamo il duro furore d'Ettore domator di cavalli,
se sfidasse da solo a solo qualcuno dei Danai
'antibion machèsasthai en ainè deiotèti'/a combattergli a fronte in tremendo duello" ${ }^{[8]}$.

E ancora più avanti è lo stesso "Ėleno, caro figlio di Priamo", a suggerire al fratello Ettore:

[6] Platone, Il convito, Garzanti, Milano, 1975, p. 201.

[7] Omero, Iliade, Libro Settimo, Einaudi, Torino, 1990, pp. 227-229.

[8] Ibidem. 
"e tu sfida i più forti degli Achei

A lottar fronte a fronte in tremendo duello" ${ }^{[9]}$.

Se si analizzano attentamente i termini utilizzati da Omero, si osserverà per esempio che antibion non può essere semplicemente un banale avverbio, in quanto il suo significato più profondo è "in faccia, fronte a fronte, corpo a corpo". Per comprendere appieno il senso basta qui soffermarci su unaltra situazione dell'Iliade in cui Omero ricorre a tale espressione, questa vola nella sua variante antibien. A tale proposito riportiamo il seguente passo dell'Iliade nel quale il saggio Nestore nel tentativo di porre fine all'ira di Achille verso Agamennone così gli parla:

"E tu non volere, Pelide, contendere col re
faccia a faccia, perché non ebbe in sorte onore comune
un re scettrato, a cui Zeus diede la gloria.
Se tu sei molto forte, una madre dea ti ha portato,
ma questi è ben più potente, ché su molti comanda"[10].

Si può osservare che qui non cè duello dal momento che si tratta di rispettare un ben preciso rapporto di gerarchia sociale, in quanto il duello si ha da fare tra persone "da pari a pari".

Si tratta in definitiva di attenersi a delle precise regole proprie al codice cavalleresco dell'antichità secondo il quale Achille non può continuare nell'alterco e magari sfidare Agamennone in combattimento perché quest'ultimo "su molti comanda" o, un po' più avanti, “...che di molti/ armati sei il capo, e Zeus t'ha affidato,/ e scettro e leggi, perché tu ad essi provveda."[11]

Il duello, quindi, non solo era una prerogativa delle élites al potere, ma esso seguiva le gerarchie interne alla stessa cerchia aristocratica, anche se il nobile superiore, compiendo oltraggio, si abbassava ad un livello inferiore: come nel caso di Agamennone nei confronti di Achille. Ed è questo uno degli elementi tipici anche del duello moderno. In effetti il carattere del rituale è di certo uno dei tratti originari conservatisi nei tempi, anche se il duello andò incontro a contaminazioni, alterazioni e anche questa stessa particolarità finirà per scomparire.

Levoluzione della società verso una visione sempre più egualitaria, come è illustrato dal Patrizi nel suo Dialogo dell'honore, il Berignano, portò sia all'accentuazione del carattere di esclusività del duello, sia alla diffusione di casi di combattimenti tra membri di ceti differenti.

Il Patrizi scrive in proposito degli studenti di Padova i quali, proprio perché studenti, si comportavano come dei cavalieri facendo "profession d"honore" "12].

Tornando allo scontro tra Ettore e il campione acheo: gli avversari di Troia non riescono immediatamente a reperire un volontario per il duello con il figlio di re Priamo. Tra i guerrieri nessuno si alza e Omero dà una descrizione rapida, ma estremamente efficace, della situazione sul campo:

[9] Ibidem.

[10] Ibidem, Libro Nono, pp. 290-291.

[11] Ibidem.

[12] Patrizi, F., La città felice. Dialogo dell'onore, il Berignano. Discorso della diversità de' furori poetici. Lettura sopra il sonetto del Petrarca. La gola, el sonno, e l'ociose piume. Per Giovanni Griffio, in Venetia, 1553. 
"quelli rimasero tutti in silenzio;

di rifiutare arrossivano e d'accettare temevano"[13].

Assai significativo è il rimprovero che segue, quello che Menelao rivolge agli Achei: in quanto, rifiutando di battersi in duello, i soldati hanno mostrato di non possedere quellautocontrollo, quel dominio delle proprie passioni e degli istinti naturali che li rende veramente uomini, essi non sono attivi/uomini nei loro stessi confronti, bensì passivi/donne e schiavi (in quanto a passività le due figure coincidono). Il tutto è racchiuso in un verso, una esclamazione di disgusto:

\footnotetext{
"Infine s’alzò Menelao e si mise a parlare

Con ingiurie e rimproveri, molto gemeva in cuore:

Ah cani da pagliaio, Achive e non Achei!

Questa sarà tremenda, tremenda vergogna,

se nessuno dei Danai muoverà incontro ad Ettore."[14]

(Estratto a sorte, sarà Aiace Telamonio a combattere).
}

Essenza quindi del duello, e sua imprescindibile condizione, è il dominio su se stessi, individuato da Foucault, e questa caratteristica fonda l'onore del singolo. Dunque non esercizio di violenza, quanto attuazione e manifestazione di una razionalità e di un ordine di cui pochi erano capaci e in virtù dei quali tali kalòi kài agathòi, tali àristoi rivendicavano il potere politico. Il duello, come prodotto umano, nasce in un ben preciso contesto e in una particolare cultura e ne esprime le linee di fondo e il pensiero. Nel nostro caso specifico si tratta della Grecia classica, caratterizzata essenzialmente dalle teorie autocratiche umane che successivamente trovarono perfetta espressione nella stessa antropologia platonica.

Se, d'altra parte, spostiamo la nostra attenzione al già menzionato libro di Massimo Cacciari Geo-filosofia dell'Europa, vi troveremo evidenziato l'importante nesso esistente tra lestetica esistenziale della Grecia antica e il duello come suo momento esplicativo, che ci permette di parlare di una sua "estetica".

A tale proposito penso che basti concentrarsi sulla terza parte, Il duello, del terzo capitolo, intitolato Gli eroi, e in particolar modo sulla critica che il Cacciari rivolge alla tesi che la studiosa Simone Weil espresse in, come si esprimerà lo stesso Cacciari, "uno dei suoi saggi più straordinari, L'Iliade, poema della forza ${ }^{[15]}$." Dove l'autrice ritiene che il poema omerico rappresenti un mondo dominato dalla bia, ovvero dalla forza, nel senso di violenza, che nel suo manifestarsi cancella l'umano e lo trasforma in qualcosa di indefinito.

Con questo l'autrice vuole, in definitiva, sottolineare l'irrazionalità, l'insensatezza di quella pratica ritenuta propria di tutti i popoli primitivi abituati alla guerra e in particolar modo della classe aristocratica incapace di sopravvivere nelle società antiche senza dare peso sociale a certi costumi e pratiche estreme come il duello o l'iniziazione.

Tali giudizi corrispondono alla realtà? È giusto affermare che nel mondo omerico

[13] Omero, Iliade, Libro Settimo, Einaudi, Torino, 1990, p. 231.

[14] Ibidem.

[15] Weil, S., "La Grecia e le istituzioni precristiane”, in: Cacciari, M., Geo-filosofia dell'Europa. Capitolo 3. Gli eroi, Adelphi, Milano, 1994, p. 95. 
e in particolar modo nel duello d'onore regnino solamente l'incoscienza e la prepotenza? Il Cacciari ritiene che se la Weil avesse senza pregiudizi di sorta rivolto il proprio sguardo al duello come viene espresso nell'Iliade, avrebbe certamente e senza ombra di dubbio scorto quell'armonia e quella misura, cioè quel lògos, quella pace suprema che il giudizio delle armi porta con sé.

Anche perché certamente si sarebbe accorta che non si tratta solamente di bia ma altresì, se non anche in prevalenza, di kràtos il vero protagonista dellopera omerica, in quanto si tratta di una volontà intelligente, di quel tipo di forza che vince l'impulso selvaggio e si autodomina. In tal senso leroe greco vive in se stesso, al proprio interno il pòlemos cosmico tra ordine e disordine, tra limite e illimitatezza, conquistando con molta difficoltà e non sempre l'armonia, l'autocontrollo che lo rende partecipe sia della natura umana che di quella divina.

Nestore ne è fulgido esempio assieme a tutti gli altri personaggi che rappresentano l'ideale greco di vita buona, di temperanza: come potrebbe l'eroe, cessare ogni scontro, posare momentaneamente le armi e acconsentire al recupero dei compagni caduti. Cosa che accade al termine del cruento duello tra Ettore e Aiace ${ }^{[16]}$, riportato dallo stesso Cacciari nella terza parte del terzo capitolo del suo libro ${ }^{[17]}$, come esplicito esempio che dimostra come scopo e condizione del duello nella Grecia antica era un qualcosa di nobile e di sentito da tutti gli individui valorosi e non il risultato del furore cieco di un insensato combattimento. Perché, altrimenti, come spiegare il fatto che Achille, alla fine del poema ${ }^{[18]}$, senza indugio rende al supplice vecchio re di Troia Priamo le spoglie del defunto figlio se in lui il nòos non prevalesse e non dominasse gli istinti?

Nel duello i due avversari stanno soli, l'uno di fronte all'altro (l'eroe è sempre solitario), si confrontano entrambi decisi a combattere sino allo stremo delle forze, ma non perdono mai il rispetto reciproco, in quanto essi costituiscono un'unità inscindibile, mai sopraffatti dal loro thymòs e dalle cieche passioni che spingono a lottare, mai dimentichi del limite rappresentato dalla Notte, di fronte al cui sopraggiungere gli eroi si lasciano persuadere alla philia, perché così è buono. In questo è da ricercare il nesso tra il duello e la grecità.

Tra l'altro, cè da dire che prima dello scontro tra Ettore e Aiace-Telamonio cè nell'Iliade un altro duello che, anche se apparentemente identico, in realtà è quanto mai diverso sia per i protagonisti che vi prendono parte, per quel che riguarda l'onore dell'eroe, ma altresì come esito e significato per le sorti della guerra di Troia ${ }^{[19]}$. I due eserciti ormai si fronteggiano e ci aspetteremmo un attacco in forze, quando viene invece annunziato un duello come soluzione ad un conflitto che pareva non aver mai fine. Si tratta del duello tra Paride e Menelao.

Ma che esito avrà tale combattimento?

Paride che invece di guardare agli interessi della res publica, di Troia, un tempo cedette per amore di una donna cioè, di Elena, per la quale egli non fu capace di contenersi. Allo stesso tempo protetto e vittima di Afrodite Pandemia, ossia della dea della gente qualsiasi, degli inferiori che non si curano di onore e gloria, che non tendono

[16] Omero, Iliade, Libro Settimo, Einaudi, Torino, 1990, pp. 227-254.

[17] Ibidem, pp.100-101.

[18] Ibidem, Libro Ventiquattresimo, pp. 843-889.

[19] Ibidem, Libro Terzo, pp. 89-116. 
a ciò che è perfetto e superiore, comè l'anima dell'uomo, come al contrario fanno gli amanti di Afrodite Urania. In definitiva, tenendo conto di tutto ciò, può egli essere valoroso guerriero, può gioire all'idea di un duello tra pari? Assolutamente no, anzi, egli, intento a lucidare la propria armatura, indietreggia impaurito, per poi, sbeffeggiato e rimproverato da Ettore, accettare la sfida.

A priori si può dire quale sarà l'esito della scena: Menelao, in netto vantaggio sull'avversario, sta per finire Paride-Alessandro strangolandolo con la cinghia dell'elmo e se non intervenisse Afrodite di certo egli sarebbe destinato a soccombere. Come può essere capace di vincere gli altri in duello chi non è capace di vincere il pòlemos contro se stesso? Non è forse questo il senso profondo della delfica prescrizione "conosci te stesso"?

I detrattori dellorigine greco-romana del duello moderno sostengono pure che i casi di combattimento tra singoli guerrieri che riempiono le pagine omeriche non costituirebbero un antesignano del duello moderno in quanto quest'ultimo vede come essenziale la presenza dei secondi, ossia delle persone fidate che facciano da testimoni e che garantiscano il rispetto di tradizioni e norme. È quanto mai sprovveduto ritenere che per la classicità greca non si possa parlare di duelli veri e propri a causa dell'assenza di secondi.

Non ci si vuole rendere conto che si tratta di un diverso mondo culturale dove il duello tra singoli individui coinvolgeva l'intera comunità sociale. Ecco perché ai combattimenti tra gli eroi omerici sono sempre presenti degli oràontes, custodi di tradizioni e testimoni della gloria e dellonore dei contendenti. Si tratta della comunità: dalle mura della città di Troia e dalle file dell'esercito acheo la collettività sta a guardare, non partecipa se non con adeguati incoraggiamenti, richiamando di volta in volta il guerriero all'onore.

E lo stesso Ettore nel fatale scontro con Achille non potrà ricorrere al soccorso attivo del proprio testimone, in quanto egli è un phàsma, un'illusione. Come abbiamo più su detto si tratta di secondi diversi da quelli dell'età moderna, ma non si può negare la loro presenza, tanto che il filosofo S. Kierkegaard in Timore Tremore ne fa un tratto distintivo tra l'eroe classico e le sue imprese e l'eroe cristiano: mentre Abramo nel sacrificare il proprio figlio Isacco è solo di fronte a Dio, non ha nulla e nessuno a incoraggiarlo e sostenerlo, nulla su cui fondarsi tranne la propria fede, Agamennone ha alle proprie spalle la collettività come testimoni, non è mai lasciato a se stesso e in caso di rifiuto egli sarebbe coperto di infamia, perché mostrerebbe di anteporre l'interesse particolare a quello universale. In definitiva questo è l'eroe classico, l'archetipo del duellante moderno.

A questo punto non ci resta che concludere questa parte introduttiva del duello europeo basandoci sulle tesi di uno dei più autorevoli studiosi del duello dell'età moderna, Brantôme ${ }^{[20]}$, il quale sostiene che il duello dell'età moderna nasce nell'Italia del XVI sec., la quale, oltre ad essere patria dei personaggi più esperti e bravi in quell'arte, lo era anche di tanta letteratura tecnica in materia.

Secondo il Brantôme, degno di menzione è certamente il magnifico trattato di Girolamo Muzio, Il Duello ${ }^{[21]}$, pubblicato a suo tempo a Venezia nel 1685, che fu la più celebre opera sull'escrime (il combattimento con la spada, la scherma), antenato della

\footnotetext{
[20] Si tratta di Pierre De Bordeille Seigneur de Brantome che nella sua opera Discours sur les duels, ritiene che i francesi avessero appreso la procedura del duello a Napoli. In De Brantome, Ouvres, La Haye 1740, pp. 110-113; 229-230.

[21] Mutio Iustinopolitano, Il duello. Con le risposte cavalleresche, Venetia, 1685.
} 
meticolosa e assolutamente esauriente trattazione contenuta nell'Encyclopédie di Diderot e D'Alembert.

Tali affermazioni di Brantôme circa l'origine italica del duello ci porta a chiederci qual era il sostrato e la corrente culturale dominante alla quale il Muzio - come del resto molti altri autori, compresi i detrattori del calibro del nostro Patrizi - attinse e di cui era manifestazione. Si tratta dell'umanesimo rinascimentale: cercare di definire tale movimento è alquanto difficile, poiché si corre il rischio di cadere nei dualismi uomonatura, letteratura-scienza, erudizione-indagine che tanto impedirono una corretta analisi dei secoli XV e XVI italiani.

Anche perché l'umanesimo non è semplicemente un movimento letterario incentrato sugli studi di grammatica, retorica, poesia, filologia e di filosofia morale.

Questo è uno sguardo parziale e riduttivo, incapace di cogliere quella rivoluzione del pensiero che sta alla base della riscoperta tanto dei testi già conosciuti nel medioevo, quanto dei manoscritti mai comparsi prima nelloccidente latino. Così, sullo stimolo dei nuovi interrogativi che animavano gli eruditi italiani, fu fatta luce sul mondo antico.

Ed è proprio questo riprendersi parte della propria storia antica la chiave di volta della nostra ricerca, in quanto permette di dire che la paternità greca del duello da noi sostenuta non si fonda su elementi estrinseci, per cui si tratterebbe soltanto di una somiglianza tra aspetti secondari, accidentali. Ma dalla certificazione che il duello moderno, anche se dimenticato per un periodo di tempo, deriva dalla Grecia classica e ne incarna la visione autocratico-platonica dell'uomo, proprio perché la sua culla è l'Italia umanistico-rinascimentale che nasce sotto il segno di Platone.

Non si tratta qui di asserzioni prive di fondamento. Basta rendersi conto che il '300 fiorentino si conclude col Petrarca che esalta la grandezza di Platone di contro allo strapotere aristotelico e il '400 si apre all'insegna del grecista Crisolora e della sua traduzione dal greco al latino della Repubblica platonica in collaborazione con Uberto Decembrio. Fu solo l'inizio: seguirono prima la traduzione di Pier Candido Decembrio, poi quella del Cassarino e infine quella dell'intero corpus platonico grazie a Marsilio Ficino (incaricato da Cosimo il Vecchio, tutto teso alla creazione dell'Accademia Platonica concepita su ispirazione del novello Platone, nella persona di Giorgio Gemisto Pletone) ${ }^{[22]}$.

Tale filosofo assegna un ruolo chiave al Simposio di Platone e il suo commento al dialogo platonico - si tratta del trattato noto come El Libro dell'Amore - ripropone con enorme successo nell'Italia del '400 le teorie platoniche dei due amori e della superiorità dell'anima sul corpo. Da evidenziare inoltre che la famosa categoria dell'amor platonico è coniata da lui ed è indispensabile per comprendere sia Dante sia Petrarca.

L'importanza di El Libro dell'Amore è ben evidente se si pensa che le più accanite critiche saranno quelle di Giovanni Pico della Mirandola, amico nonché avversario di Ficino, aristotelico averroista di formazione e grande conoscitore di Platone.

A tale proposito, nella prolusione alla purtroppo famosa discussione romana, l'Oratio, trattando del ritorno dell'uomo a Dio in virtù della propria facoltà imitativa, il regenerari in superiora, egli scrive:

[22] Garin, E., Il ritorno dei filosofi antichi, Bibliopolis Editore, Napoli, 1994. 


\begin{abstract}
"Chi mai vorrà toccare le scale del Signore o con piede impuro o con mani non monde? [...] Ma quali questi piedi e queste mani? Piede dell'anima è senza dubbio quella parte vivissima con cui si appoggia alla materia come alla terra; la facoltà [...] fonte di libidine e maestra di mollezza sensuale. E mani dell'anima perché non chiamare la facoltà irascibile, che, a servizio degli appetiti, per essi combatte e si slancia [...]. [...] tutta la parte sensibile in cui hanno sede le lusinghe corporee che, come dicono, tengono l'anima per la gola, laviamo con la morale [...]. Ma neppur questo basterà $[\ldots]$ se prima non saremo stati ben preparati e istruiti $[. .$.$] con la parte discorsiva$ o raziocinante dell'anima"[23].
\end{abstract}

Ebbene questo è il contesto in cui nasce anche la trattatistica del duellare moderno come altresì della sua più valida negazione patriziana ${ }^{[24]}$. Per questi motivi è più che ovvio che esso non può che essere imbevuto di platonismo e delle teorie greche del dominio di istinti e passioni che l'uomo, per essere attivo, deve esercitare su se stesso, che per quell'antropologia superlativa che caratterizza il pensiero di Pico della Mirandola questo vuol dire affermare la suprema libertà che fa dell'uomo una creatura sui generis nell'universo: superiore tanto agli animali quanto agli esseri angelici. Da intendersi che la filosofia morale di cui si parla altro non è che l'askèsis greca e il suo fine ultimo è rendere l'uomo phrònimos, ossia temperante tra furore e pudore.

Da chi potevano trarre la propria origine quella perfezione dei gesti del duello, il merito individuale e l'onore che si conquista solamente con la virtù e le prodezze individuali? A tale proposito Jean-Pierre Labatut del cavaliere dice che:

"egli si batteva con ardore, ma senza annientare il vinto di cui rispettava i pregi. Sicuramente i comuni mortali non potevano capire perché si rendessero gli onori ad un nemico defunto. [...] La nobiltà possedeva la virtù «aristocratica», un insieme di doti morali che [...] l'italiano Muzio, nel secolo XVI, faceva consistere [...] nel coraggio, nella lealtà, nella fedeltà, nel disinteresse, nella generosità" $^{[25]}$.

Come poteva il cavaliere essere capace di tali virtù, gesta e disprezzo della morte se non dopo aver vinto se stesso, oppure, usando un'impareggiabile espressione di Pico, dopo aver acquietato "gli sfrenati tumulti della bestia multiforme e le minacce e la rabbia e l'impeto del leone" e instaurato "fra la carne e lo spirito un inviolabile patto di santissima pace"[26].

Non dimentichiamo in ultimo che il trattato che esprimeva la nuova situazione rinascimentale, sociale e politica e che aveva lo scopo di fornire alle nuove aristocrazie di corte un modello di vita e di comportamento, Il Cortegiano di Baldesar Castiglione, vede impianto, struttura e conclusione platonici: in particolare egli parla di un seme di virtù incluso e sepolto nell'anima, che il soggetto stesso, come un agricoltore-demiurgo, fa germogliare e fruttificare, simbolo della bellezza interiore, quale ideale che traluce nel mondo e nella realtà sensibile.

Se ne deduce che il comportamento raffinato, misurato e composto di colui che popola le corti sia il prodotto di un animo altrettanto raffinato e disciplinato, ovvero cortese.

[23] Giovanni Pico della Mirandola, Oratio de hominis dignitate, a cura di Garin, E., Edizioni Studio Tesi, Pordenone, 1994.

[24] Patrizi, F., op.cit.

[25] In Labatut, J.P., Le nobiltà europee dal XV al XVIII secolo, Il Mulino, Milano, 1999, p. 24.

[26] Giovanni Pico Della Mirandola, ibidem. 
Purtroppo tanto il platonismo quanto l'arte del duellare che ne era l'incarnazione in quanto dominio della parte oscura della propria anima, andarono incontro a volgarizzazione, a un lento declino che fece loro perdere l'originario senso e valore e li rese mode e paraventi passibili delle critiche del Patrizi in quanto mescolava l'onore con semplici sbaruffate tra studenti. Ma questa è solo la fine del duello per ragion d'onore.

\section{Dell'onore e del duello nel Rinascimento}

Il duello rinascimentale, quale singulare certamen ${ }^{[27]}$, rappresenta un istituto che, anche se "polimorfo per definizione", fu comunque in grado storicamente "di cogliere inflessioni di enorme raffinatezza e sottigliezza, nonché di rimodulare assai variamente funzioni e struttura in differenti contesti sociali e culturali" ${ }^{28]}$.

Per esaminare in che modo e attraverso quali forme questa istituzione abbia dato un contributo essenziale nella costruzione dell'uomo moderno diventando, a suo tempo, "una delle essenziali chiavi di volta del medioevo e dell'età moderna in Europa" ${ }^{[29]}$, è quanto mai necessario rivolgere brevemente la nostra attenzione non solo sul duello per punto d'onore ma altresì su altre due tipologie di duello in auge nel medioevo, ovvero sul duello giudiziario per prova della verità e sul duello in torneo per ostentazione di forza e di valore.

Per quanto riguarda il duello giudiziario per prova della verità o ordalico, di monomachia e di pugna ${ }^{[30]}$ si deve dire che nasce indipendentemente dalla tradizione greca, ancora sconosciuta in Europa visto che ci troviamo nel medioevo, e come tale è il primo tipo di duello ad apparire nell'Europa cristiana. A dire il vero questa tipologia di duello era praticata già in epoca remota presso alcune popolazioni dei territori centro-settentrionali, e si diffuse anche nelle regioni mediterranee dopo la disgregazione dell'Impero romano d'occidente, conservando quasi del tutto inalterata l'originaria matrice germanico-barbarica.

La monomachia, che in età feudale era riservata principalmente ai milites et nobiles, "consisteva in un giudizio di Dio concesso dal giudice per risolvere liti civili e criminali" ${ }^{[31]}$ dove la sentenza definitiva dipendeva dall'esito dello scontro fra uomini armati. In tal senso, il duello giudiziario era l'espressione più evidente "di una civiltà giuridica in cui le dimensioni della religione, della morale e del diritto venivano percepite insieme ed indistintamente nell'unità del verbo divino" ${ }^{[32]}$.

Le diverse trattazioni in materia ci permettono di concludere che i duelli giudiziari ordalici si svolgessero secondo le consuetudini locali, attenendosi comunque a degli universali principi d'onore personale e di rispetto per l'avversario in quanto "la fraternità cavalleresca dei duellanti tendeva a favorire lo sviluppo di istituti unitari: la cavalleria era ceto ed istituzione largamente sovranazionale" ${ }^{[33]}$.

Al duello giudiziario si poteva accedere o tramite la decisione del giudice

[27] Cavina, M., Il sangue dellonore. Storia del duello, Laterza, Roma-Bari, 2005.

[28] Ibidem, p. 6.

[29] Ibidem, Preludio, p. VII

[30] Si trattava di diversi tipi di prove quali: del fuoco, dellacqua fredda, del ferro arroventato, delloffa, della bilancia, ed altro ancora dipendentemente dalle culture socio-territoriali.

[31] Cavina, M., op. cit., p. 6.

[32] Ibidem, p. 8.

[33] Ibidem, p. 27. 
o tramite la semplice richiesta/sfida dell'accusatore senzalcuna precedente attività giudiziale.

Questo modello di duello entrò in crisi con l'affacciarsi dell'umanesimo italiano quando sulla scena europea appare l'aulico duello d'onore all'italiana ${ }^{[34]}$. Tale irreversibile declino del duello giudiziario ordalico fu principalmente causato da tre fattori: uno di tipo economico, i costi notevoli a carico delle parti; uno di tipo legale, le prime politiche accentratrici per via legislativa; uno di tipo morale, e le posizioni del mondo ecclesiastico.

In effetti cè da dire che è stata la Chiesa, tramite il diritto canonico che, a partire soprattutto dal XII secolo, si schierò in modo intransigente contro il proliferare di giudizi di Dio. Giudizi ordalici che, a dire il vero, con la Controriforma diventeranno appannaggio esclusivo della Santa Inquisizione. Cè comunque da rilevare che questo giudizio negativo della Chiesa verso il proliferare del duello, in tutte le sue tipologie più o meno cortesi, sarà costante e sfocerà, come si vedrà più avanti, nella discussione sul decreto di condanna e sulla conseguente proibizione del duello da parte del concilio di Trento.

Tralasciando le questioni di ordine economico che sottoforma di cauzioni processuali, spese d'allestimento, ed altro ancora prevalentemente riguardavano le parti in causa, cè ancora da dire che al progressivo abbandono del duello ordalico contribuì anche una serie di legislazioni secolari restrittive. Queste emersero durante quel processo di scientificizzazione della legge che, sulla base del diritto romano-canonico, puntava ad assoggettare istituti e consuetudini all'analisi e alle categorie concettuali dei giuristi dotti.

Anche se, cè da dire comunque che il duello giudiziario venne sempre percepito quale pratica sostanzialmente estranea all'universo del diritto comune, solo localmente ed eccezionalmente era ammissibile per via normativa.

Nel medioevo di pari passo con il duello giudiziario ordalico su scala europea si sviluppa altresì un altro tipo di duello, la giostra o duello in torneo per ostentazione di forza e di valore ${ }^{[35]}$, per lo più finalizzato a dare pubblica dimostrazione del coraggio e del valore marziale dei contendenti, a mantenere in allenamento quanti esercitavano il mestiere delle armi, ad esprimere in un linguaggio festoso e fastoso la complessità dell'etica cavalleresca, a trasmettere consuetudini e modelli comportamentali nobiliari.

La conseguente condanna ecclesiastica del torneo e la scomunica dei loro partecipanti, sempre apparsa tutto sommato abbastanza blanda, e il fatto che l'omicidio colposo che talvolta vi si commetteva risultasse escluso dal diritto comune, non impedirono a questo tipo di duello di entrare in crisi nel Cinquecento, una fortuna che peraltro andò declinando lentamente nel corso dell'intero antico regime. Ciò si spiega sia con il succedersi della Riforma e della Controriforma che divise l'Europa cristiana in due, e dunque di un clima poco propizio a fatui giochi di guerra, sia col verificarsi di alcuni tragici episodi che - a cominciare dalla morte in torneo nel 1559 del re di Francia, Enrico II - suscitarono unenorme impressione e presentimenti sinistri nei contemporanei.

[34] La cosa è ben documenta nel famoso Tractatus de bello, de represaliis et de duello, redatto nel 1360 da Giovanni da Legnano.

[35] Vedi a tale proposito Cardini F., Guerre di primavera. Studi sulla cavalleria e la tradizione cavalleresca, Firenze, Le Lettere, 1992; Id., L'acciar de' cavalieri. Studi sulla cavalleria nel mondo toscano e italico (secoli XII-XV), Firenze, Le Lettere, 1997; Flori, J., Cavalieri e cavalleria nel Medioevo, tr. it. di Aboaf, M. e Pico, S., Einaudi, Torino, 1999. 


\section{Il duello legale per 'punto d'onore'}

Nel '500 nobili e militari iniziarono a ricorrere al duello d'onore per "risolvere la gran parte delle loro controversie al di fuori di qualsiasi intromissione statale"[36]. Il diritto al singulare certamen era "percepito come un intangibile diritto naturale, che si legittimava nella terra e nella storia, nel sangue antico di nobili antenati e nell'integerrima osservanza delle virtù marziali" ${ }^{[37]}$.

Anche se il diritto comune e ordinario interpretava e reprimeva l'ingiuria come violazione del buon ordine pubblico, i milites et nobiles, ovvero ogni individuo di elevata condizione sociale, ritenevano che difendere lonore esterno fosse diritto/dovere di ogni gentiluomo offeso. E l'arma che più di altre si addiceva a recuperare l'onorabilità era di sicuro la spada che, in quanto simbolo cavalleresco per eccellenza, veniva a costituire il mezzo attraverso il quale purgare convenientemente l'ingiuria e "dimostrare al proprio ceto dessere ancora degno di farne parte, in quanto uomo d'onore che non si lascia 'sprezzare' od oltraggiare impunemente" ${ }^{\text {[38] }}$.

Veniva, in tal modo, dato risalto alla figura di un cavaliere intimamente persuaso di far parte di una categoria eletta. Ceto sociale le cui regole di condotta si basavano su una presunta missione che consisteva nell'attuare la giustizia naturale per una specie di vocazione innata, che aveva ereditato con il proprio sangue e che con il proprio sangue doveva saper realizzare.

Il duello per punto d'onore ebbe la sua stagione più gloriosa socialmente e più raffinata culturalmente in tutte le corti italiane del Rinascimento sottoforma di scienza cavalleresca. Questo spiega tanto perché la scienza cavalleresca, in questo periodo nel piccolo paese, come amava definire l'Italia il Patrizi, si sviluppa una ricchissima e variegata trattatistica del duello ed i molti pensatori dellepoca, tra i quali appunto il Patrizi, si confrontano tra di loro in erudite interpretazioni sul significato dellonore e sulletica della nobiltà.

Trattatistica che allestero venne percepita come un prodotto originale della cultura italiana in quanto il modello italiano di singolar tenzone, venuto alla luce presso tali corti, ebbe così grande risonanza in tutta Europa, pur non diventando mai egemone al di là delle Alpi, dove si preferiva pattuire scontri fra privati senza alcuna autorizzazione ufficiale ${ }^{[39]}$.

La complessità della trattatistica del singulare certamen, sviluppatasi nel piccolo paese, da subito presupponeva una netta distinzione tra il duello, la vendetta e la gloria. $\mathrm{Fu}$ proprio per queste ragioni, inerenti al distinguo, che il duello per prova d'onore fu strutturato come un vero e proprio processo per il ripristino dellonore infangato. Da ciò derivava che il duello in qualità di prova d'onore "non era vendetta più di quanto lo fosse una qualsiasi azione giudiziaria, formalmente diretta a veder riaffermato il proprio buon diritto"[40].

Lautorità pubblica possedeva pieno arbitrio sulla valutazione della giustificabilità o meno della causa di duello. Dove invece, il semplice accordo tra le parti sprovvisto

\footnotetext{
[36] Cavina, M., op. cit., p. 41.

[37] Ibidem, p. 46.

[38] Ibidem, p. 71

[39] In Erspamer, F., La biblioteca di Don Ferrante. Duello e onore nella cultura del Cinquecento, Bulzoni, Roma, 1982.

[40] Cavina, M., op. cit., p. 46.
} 
di concessione formale dava vita a scontri criminosi, valutati alla stregua di meri duelli alla macchia che rientravano nel novero delle volgari baruffe, delle vendette e dei privati linciaggi, e che come tali erano esposti alle regole della giustizia ordinaria dipendentemente alle varie normative locali.

Il duello giudiziario donore si plasmò attraverso la dialettica fra: i professionisti del duello, chiamati anche "professori d'onore", che fondavano le proprie concezioni sul mito dell'esperienza dei cavalieri, e i giuristi, impegnati ad esaltare il primato della giudiziarietà, in quanto strutturato come un vero e proprio processo e i cui principali esponenti erano Paride dal Pozzo (Puteo) e Andrea Alciato. Ed ecco spiegato perché, questi ultimi, che operavano anche nelle università e nei tribunali, avevano tutte le pretese di giuridicizzazione secondo il diritto comune, in quanto consideravano quanto mai essenziale subordinare la prassi alla dottrina, il factum al ius. Dove invece i professori d'onore, basti qui nominarne due nelle persone di Girolamo Muzio e di Sebastiano Fausto da Longiano, erano uomini d'arme esperti di consuetudini nobiliar-militari e che, frequentando corti ed eserciti, collocavano al centro delle proprie preoccupazioni il momento sostanziale dell'onore, anziché quello procedurale del giudizio.

Si arrivava allo scontro d'armi quando uno dei litiganti imputava formalmente allaltro di aver mentito nelle sue asserzioni ingiuriose, violando quel supremo obbligo di verità cui ogni cavaliere era tenuto per poter continuare ad essere reputato tale. Nella prassi, una delle lesioni più ricorrenti all'onore era l'accusa del mancato rispetto della parola data, inequivocabile indice di un sentito e mal celato disprezzo.

A differenza del duello giudiziario per prova della verità o ordalico, implicante l'istituto del campione (pugiles che si fronteggiavano in sostituzione delle parti lese), il duello d'onore veniva combattuto direttamente dalle parti, che dovevano essere incarnate da individui di sesso maschile, appartenenti al medesimo ceto, dotati di buona salute, che avevano l'età di portare le armi e in possesso d'integrità d'onore.

Condizioni che comunque non erano del tutto indispensabili, perché nel caso di requisiti fisici non omogenei, si provvedeva adeguatamente a sopperire a quelle manchevolezze che rendevano impari il duello tra pari. Ad esempio, bendando un occhio del contendente che non era orbo o altro ancora.

In quel periodo era diventata prassi ordinaria delle parti in causa assoldare professori d'onore e giuristi dotti in scienza cavalleresca per studiare la vertenza e definire le condizioni di ammissibilità ed eventualmente i termini del duello. Una delle questioni fondamentali era individuare il provocato, uno status che nei fatti non risultava sempre agevole da distinguere, e che, secondo la consuetudine, aveva il diritto e il privilegio di scegliere tanto il campo quanto le armi.

Fra i compiti dei giuristi si annoverava anche la stesura dei cosiddetti cartelli, la cui disciplina fu minuziosamente costruita sulla falsariga degli atti processuali ordinari. Il testo del cartello, preparato da una delle parti in causa, doveva essere sottoscritto da due o tre testimoni ed era consegnato sia al giudice del duello sia all'avversario, il quale aveva a disposizione un tempo pattuito per rispondere con un altro cartello. Si provvedeva a divulgare i termini della sfida in luoghi pubblici, di frequente con l'ausilio della stampa, affinché fosse impedita l'allegazione d'una finta ignoranza e soprattutto in modo che la nobiltà venisse a conoscere i particolari della causa del disonore. Essendo 
l'onorabilità una virtù pubblica il suo ripristino e la sua tutela non poteva che aver luogo dinanzi al tribunale dell'opinione.

I cavilli e i sottili artifici terminologici che caratterizzavano non solo i cartelli, ma anche un'infinità di altri problemi affrontati dai giuristi quali le discussioni sui termini temporali del duello e la ricerca d'un giudice imparziale, irritavano i professori d'onore, secondo i quali chi si perdeva in vuote e improduttive disquisizioni attorno a questi temi mostrava di non possedere coraggio palesando in modo inequivocabile la disonorevole volontà di evitare le armi.

Il duello d'onore rinascimentale si svolgeva all'interno di un campo franco il più delle volte circoscritto da un solco d'aratro, da uno steccato di legno, da un muro di pietre o da una lunga fune e coinvolgente due gentiluomini dotati di medesimo equipaggiamento difensivo, che talvolta poteva risultare abbastanza pesante.

Il confronto in armi era sorvegliato dal signore del campo e se il duello non era stato dalle parti precedentemente patteggiato altrimenti - al primo sangue o a le tre prime ferite sanguinanti - proseguiva ad oltranza, se necessario, dall'alba al tramonto del giorno prescelto. Se al termine del giorno pattuito non ci fossero stati risultati di sorta, si giudicava riconfermato l'onore del reo. Per non dare adito a disonoranti sospetti, la dinamica della sfida doveva dimostrare la buona voglia delle parti alle armi. Al giudice non era consentito interrompere lecitamente il duello contro la volontà delle parti, sotto pena del pagamento delle spese al provocatore; e allo stesso modo, egli risultava patrimonialmente responsabile se graziava il vinto senza il permesso del vincitore.

I patrini, padrini o avvocati dei combattenti, avevano il compito di tutelare i loro protetti in tutti i contrasti relativi non già alla causa donore, bensì ai problemi connessi con lo scontro cruento e riguardante i pareri sull'uguaglianza delle armi, sullequa disposizione nello steccato, od altro ancora. I padrini erano coadiuvati da un notaio, da un armaiolo e da diversi confidenti. In questa categoria rientravano amici o parenti dell'una e dell'altra parte il cui scopo principale era quello di stare attenti e così di contribuire in modo attivo ad evitare ogni genere di scorrettezza da parte dei contendenti.

Lo sconfitto, che si presumeva avesse combattuto ingiustamente, cedeva di solito all'avversario armi, insegne, selle, cavalli e abbigliamento. Oltre a questo aveva anche lobbligo di pagare tutte le spese del vincitore, che potevano essere anche assai consistenti, e inoltre diveniva suo prigioniero di fede. A questo punto, il vincitore lo donava al signore del campo oppure lo teneva presso di sé come una sorta di servo, almeno finché non veniva pagato un riscatto in cambio della sua libertà.

E se succedeva che una parte non si presentava in campo entro il tramonto della giornata convenuta, la sua causa veniva dichiarata ingiusta dal giudice e dai suoi consiglieri, onde egli - come reo confesso - era diffamato e interdetto da qualsiasi altro duello per prova d'onore. In maniera analoga, l'ammissione del proprio torto resa pubblicamente nella confessio contraria, detta anche disdetta, ottenuta nella maggior parte dei casi quando allavversario vinto veniva posta la punta della spada alla gola, il che equivaleva alla morte civile del cavaliere. In genere, secondo l'etica del duello cavalleresco, questa era la sorte più spaventosa che potesse occorrere ad un uomo nobile. 


\section{La 'rappacificazione' e il duello 'clandestino'}

La ferma condanna tridentina ${ }^{[4]]}$ e una legislazione secolare via via più ramificata e impetuosa nelle sue mire razionalizzatrici e, dunque, ostile alle pratiche giudiziarie consuetudinarie, comunitarie e negoziate prodotte autarchicamente dalla società, trasformarono lormai diffusissima tenzone solenne per punto donore in procedura contra legem. Tuttavia, né la vasta campagna pubblicistica contro il singulare certamen, né le intransigenti prese di posizione della Chiesa cattolica, né le politiche repressive - peraltro, non sempre adeguate alla situazione - delle autorità secolari, riuscirono a contenere l'aumento esponenziale del numero di scontri d’armi clandestini, cioè di duelli d'onore contrattati fra le parti prescindendo dalla gravità della causa in gioco e, dunque, sprovvisti dell'autorizzazione ufficiale del potere pubblico.

$\mathrm{Da}$ canto suo, questa nuova situazione venutasi a creare comportava un gran risparmio di tempo e di denaro, anche perché in tal modo venivano eliminati diversi fattori inerenti alla tipologia del processo giudiziario. Così per esempio non si dava più importanza a rilevare l'adeguatezza della domanda e la concessione di campo franco, non si prevedeva alcun giudice, il numero dei cartelli era ridotto al minimo, non si ricorreva ai pareri d'onore e si duellava a piedi, armati d'una spada e indossando una semplice camicia in luogo dei tradizionali equipaggiamenti difensivi.

Nondimeno, il duello clandestino si accollava due notevoli inconvenienti per entrambe le parti. Come primo la perseguibilità penale, a cui si riusciva nondimeno $\mathrm{ad}$ ovviare di frequente sia per l'omertà determinata dalla solidarietà di classe, sia per la grazia che interveniva immediatamente o in seguito ad una breve contumacia; e come secondo l'ardua difficoltà di definire le eventuali conseguenze del rifiuto d'una sfida a duello. E, naturalmente, al reo-provocato non erano più riconosciuti i diversi privilegi accordati nel duello lecito iniziando dalla scelta delle armi e del campo alla tutela contro possibili agguati e scorrettezze da parte dell'attore-provocante.

Il parziale declino del controllo politico e giudiziario sulla vita dei gentiluomini veniva a rafforzare la ristrettezza del codice d'onore in merito al processo del singular certamen, che in tal modo aveva modo di esprimersi in tutta la sua sottigliezza negli ambiti e nelle situazioni più disparate, trasportandosi in tal modo nelle più diverse competizioni socio-culturali in voga in quel periodo storico ed in quella data regione, come per esempio durante il gioco e durante la danza, durante la messa e durante la guerra, per amore o per capriccio.

Nella seconda metà del Cinquecento la letteratura d’onore si convertì in blocco alla trattatistica sulle cosiddette paci private, assecondando in tal modo, anche se solo esteriormente, il nuovo rigorismo controriformista. In questi scritti la pace negoziata fra privati o altrimenti detta rappacificazione, era dipinta come opera giusta, in sintonia con l'ordine pubblico e con l'etica cristiana che non permetteva spargimenti di sangue tra cristiani. Anche se, a dire il vero non si mancava contemporaneamente di discorrervi ancora animatamente del duello, e questo al riparo dagli strali ecclesiastici e dietro lo

[41] A proposito dell'atteggiamento della Chiesa cattolica intorno al duello donore, e in particolar modo sulla bolla Ea quae (1560), sul testo tridentino (1563) e sulle loro ripercussioni vedi Angelozzi, G., La proibizione del duello: Chiesa e ideologia nobiliare, in Prodi, P. e Reinhardt, W. (a cura di), Il Concilio di Trento e il moderno, Atti della XXXVIII settimana di studio (Trento, 11-15 settembre 1995), Bologna, Il Mulino, 1996, pp. 271-308. 
scudo d'un titolo pacifista ${ }^{[42]}$. La figura cruciale di questo nuovo tipo di trattatistica era il cavaliere che si proponeva come arbitro-pacificatore di vertenze d'onore.

$\mathrm{Si}$ deve sapere, infatti, che la rappacificazione non era quasi mai trattata dai soggetti coinvolti personalmente, in quanto questo era il compito del cosiddetto mezzano. Si trattava di un gentiluomo autorevole ovvero di un amico delle parti che interveniva dipendentemente dalla situazione incresciosa venutasi a creare tra i due contendenti con il compito di trovare una soluzione onorevole per ambedue i litiganti e adeguata alle questioni cavalleresche senza addivenire ad uno scontro d'armi.

Non solo gli abbattimenti clandestini rimasero a lungo tollerati nell'Europa meridionale e specialmente in Spagna e in Francia ${ }^{[43]}$, ove essi erano già da lungo tempo praticati. Da dove, nel tardo Cinquecento, sbarcarono e si diffusero rapidamente sia in Inghilterra che in Irlanda, per poi di seguito passare in Germania, in Polonia, in Ungheria e nei Paesi scandinavi ${ }^{[44]}$, per penetrare, alla fine del XVII secolo, anche nella Russia degli zar ${ }^{[45]}$.

\section{Riflessione conclusiva della prima parte}

Per terminare questa parte referenziale dedicata al duello per prova donore cè da dire ancora che dappertutto l'abbandono del duello iniziò con la comparsa della nuova formazione borghese della società europea borghese e senza classi, preparata dall'Illuminismo. In questa nuova direzione sociale, verso la quale andava la società europea, un precoce esempio del nuovo orientamento culturale fu certamente il libro di Scipione Maffei intitolato Della scienza chiamata cavalleresca (1710), con il quale l'autore negava recisamente qualsiasi fondatezza razionale alla scienza dell'onore nobiliare contrapponendo a questa vulgata tradizionale, pomposamente rappresentata in quegli stessi anni da una sterminata opera in fieri, che rimase però incompiuta e riguardante l'Ateneo dell'uomo nobile, di cui Agostino Paradisi senior riuscì a pubblicare - fra il 1704 e il 1731 - soltanto i primi cinque volumi. Dove invece l'ottavo, che avrebbe dovuto trattare in modo esauriente del duellare, non fu mai pubblicato ${ }^{[46]}$.

Idee illuministiche che, da lì a pochi decenni, a macchia d’olio si propagarono in buona parte d'Europa con la convinzione sempre più ferma che il sovvertimento radicale della società e dei suoi valori avrebbe fatalmente cancellato anche i duelli di ogni tipo e altresì il punto d'onore ${ }^{[47]}$. In ambito italiano e non solo, il capostipite di quest'aria culturale rivoluzionaria fu il trattato di Cesare Beccaria, Dei delitti e delle pene $(1764)^{[48]}$.

[42] In effetti, un qualcosa di simile a quello che avviene oggi nel campo degli interventi militari occidentali nelle diverse parti del mondo sotto forma di azioni di pace, interventi umanitari, ed altro ancora.

[43] Chauchadis, C., La loi du duel. La code du point d'honneur dans l'Espagne des XVI $I^{e} X V I I^{e}$ siècles, Toulouse, Presses Universitaires du Mirail, 1997; Brioist, P., Drévillon, H. et Serna, P., Croiser le fer. Violence et culture de lépée dans la France moderne (XVI ${ }^{-}$-XVIII ${ }^{e}$ siècles), Seyssel, Champ Vallon, 2002.

[44] E questo soprattutto grazie alla guerra dei trent'anni (1618-1648).

[45] Reyfman, I., Ritualized Violence Russian Style. The Duel in Russian Culture and Literature, Stanford, Stanford University Press, 1999. [46] Per saperne di più sul celebre erudito e uomo di lettere veronese Scipione Maffei (1675-1755), si consiglia: Scolari, A., Il Consiglio Politico di Scipione Maffei in "Atti e memorie dell'Accademia di agricoltura, scienze e arti di Verona", a. IX (1932), pp. 37-87. Poi Donati, C., Scipione Maffei e la 'Scienza chiamata cavalleresca'. Saggio sull'ideologia nobiliare al principio del Settecento in "Rivista storica italiana", a. XC (1978), pp. 30-71. E ancora Romagnani, G. P., Scipione Maffei e il Piemonte, in "Bollettino storico-bibliografico subalpino", a. CII (1986), pp. 133-227; Id. (a cura di), Scipione Maffei nell'Europa del Settecento, Verona, Consorzio Editori Veneti, 1998, pp. 205-237.

[47] Anche se, a dir il vero, il clima di ostilità nei confronti del singolar certame si attenuò molto nel corso della Rivoluzione francese, allorché diventarono assai frequenti i duelli per motivi politici. In seguito, le armate napoleoniche e lo spirito del bonapartismo diffusero il senso dellonore - specie nei ranghi degli eserciti - in quasi tutto il Vecchio Continente, e il duello visse un periodo di ripresa. La Restaurazione ne riconfermò pienamente l'importanza in seno alla società europea, anche se si praticavano più che altro duelli fra militari. [48] Beccaria, C., Dei delitti e delle pene, Mondadori, Milano, 2002. 


\section{BIBLIOGRAFIA}

ARCARI, P. M., 1935, Il pensiero politico di Francesco Patrizi da Cherso, Zamperini e Lorenzini, Roma.

BECCARIA, C., 2002, Dei delitti e delle pene, Mondadori, Milano.

BRIOIST, P., DRÉVILLON, H., et SERNA, P., 2002, Croiser le fer. Violence et culture de l'épée dans la France moderne (XVI ${ }^{e}$-XVIII siècles), Seyssel, Champ Vallon.

CACCIARI, M., 1994, Geo-filosofia dell'Europa, Adelphi, Milano.

CARDINI, F., 1992, Guerre di primavera. Studi sulla cavalleria e la tradizione cavalleresca, Le Lettere, Firenze.

CARDINI, F., 1997, Lacciar de' cavalieri. Studi sulla cavalleria nel mondo toscano $e$ italico (secoli XII-XV), Le Lettere, Firenze.

CASTELLI, P., 2002, Francesco Patrizi, filosofo platonico nel crepuscolo del Rinascimento, Leo S. Olschki editore, Firenze.

CAVINA, M., 2005, Il sangue dellonore. Storia del duello, Laterza, Roma-Bari.

CHAUCHADIS, C., 1997, La loi du duel. La code du point d'honneur dans l'Espagne des XVI ${ }^{e}-X V I I^{e}$ siècles, Presses Universitaires du Mirail, Toulouse.

DE BORDEILLE, P., 1740, Discours sur les duels, De Brantome, Ouvres, La Haye.

DONATI, C., 1988, Lidea della nobiltà in Italia: Secoli XIV-XVIII, Laterza, Roma-Bari.

ERSPAMER, F., 1982, La biblioteca di Don Ferrante. Duello e onore nella cultura del Cinquecento, Bulzoni, Roma.

FLORI, J., 1999, Cavalieri e cavalleria nel Medioevo, tr. it. di M. Aboaf e S. Pico, Einaudi, Torino.

GARIN, E., 1994, Il ritorno dei filosofi antichi, Bibliopolis, Napoli.

KIERNAN, G. V., 1999, Il duello. Onore e aristocrazia nella storia europea, Marsilio, Milano.

LABATUT, J. P., 1999, Le nobiltà europee dal XV al XVIII secolo, Il Mulino, Milano.

MIRANDOLA, G. P., 1994, Oratio de hominis dignitate, a cura di Eugenio Garin, Edizioni Studio Tesi, Pordenone.

OMERO, 1990, Iliade, Einaudi, Torino.

PLATONE, 1975, Il convito, Garzanti, Milano.

PRODI, P., REINHARDT, W., (a cura di), 1996, Il Concilio di Trento e il moderno, Atti della XXXVIII settimana di studio (Trento, 11-15 settembre 1995), Il Mulino, Bologna.

REYFMAN, I., 1999, Ritualized Violence Russian Style. The Duel in Russian Culture and Literature, Stanford University Press, Stanford. 


\section{DVOBOJ U RAZDOBLJU RENESANSE}

U ovom ćemo se kratkom prikazu osvrnuti na razdoblje između druge polovice 15. i prve polovice 16. stoljeća jer se ozbiljna i jasna svjedočanstva o dvoboju mogu pronaći upravo u tom periodu i to istovremeno u književnim djelima te u djelima koja se bave teorijom dvoboja. Što se djela teoretičara dvoboja tiče, osim velikoga broja eseja, valja svakako uzeti u obzir i značajan broj njihovih izdanja, posebice onih najpoznatijih. U njima će se, naime, pored nezaobilaznoga sjedinjenja interesa između epskih pjesnika i teoretičara dvoboja, pojaviti i sličnosti u samom terminološkom određivanju: i jedni i drugi smatraju da je dvoboj "dokaz" krajnjega ili završnoga suda. Takva supstancijalna sličnost u definiranju dvoboja ne mora nužno dovesti do međusobnoga približavanja filozofskih uvjerenja jer se literarni dvoboj razlikuje od realnoga upravo po svom filozofskom poimanju pa će se pokušati osvijetliti problematike koje su nastale kao posljedica institucije dvoboja $u$.

Dvoboj se, naime, prevodio u socijalno pitanje velikoga značaja uslijed svoje dvojake konotacije: kao "božji sud", odnosno kao specifičan oblik odnosa svjetovnoga i svetoga, i kao "inculpata tutela", odnosno kao specifičan oblik posezanja za nasiljem u svrhu očuvanja temeljnoga prirodnoga prava. U oba je slučaja dvoboj shvaćen kao izraz časti, bilo individualne ili plemićke, o čijoj će utemeljenosti progovoriti Petrić. Iz te se definicije časti, o kojoj Petrić progovara u platonskome duhu, mogu iščitati politička pitanja značajnoga dosega u kojima se na različite načine progovara o pitanju odnosa između čovjeka i Države. Uključivši se u širu raspravu vezanu za uporabu nasilja i za razgraničenje između privatnoga i javnoga nasilja, između osvete i "bellum iustum", dvoboj je kao oblik/izraz časti poprimio značajne socijalne i političke konotacije.

Ključne riječi: teorijska tumačenja dvoboja, "pravedni" razlog, dvoboj kao "dokaz", Platon, Ilijada, vrline, etika

\section{THE DUEL IN THE RENAISSANCE}

The duel has always represented an institution subject to very strict rules in the way of using strength not to prove who is the strongest and the most skilful, but as a test of virtue (legal, ethical, metaphysical, sports and so on) able to reveal a kind of superior judgement, in any case always based on the virtuosity of the contestants. A typical example is represented by the judicial duel. We can, therefore, define the "duel" as the mirror or the revealing instrument of the "nature" of the "knight". This short essay refers particularly to the period of time between the second half of the 15th and the first half of the 16th century, since it is in this period that we can find serious and clear evidence about duels. Regarding works of dogmatic teaching, besides the high number of treatises we should take into consideration also the numerous new editions, especially the most famous ones. Here, besides the inevitable community of interests 
between epic poets and writers of treatises, also a substantial terminological identity will be noticed - for example, both poets and writers of treatises think that the duel is a «test» of final judgement. This does not involve at the same time convergence of philosophical convictions, since, as it will be seen, the literary duel differs from the real one precisely in different understanding of fighting. Thus, considering on the one hand the different conceptions as characteristics peculiar to the literary duel, and on the other, to the real one, the present essay aims to explain the problems these institutions themselves implied in the considered historical period of time.

In both cases it was about a kind of honour, individual and nobiliary, of which Patrizi will discuss the validity, especially in terms of virtue. In this definition of honour - which Patrizi faces with platonic taste - we can identify matters of high political significance, which claimed, in various forms, the subject of the relationship between the individual and the State. In this way the duel of honour assumed a decisive aspect in terms of social and political connotations, entering the larger debate which includes the problem of the use of violence and the boundary between private and public violence, between feud and bellum iustum.

Key words: treatises on duels, the 'good' cause, the duel as a 'test', Plato, the Iliad, ethics, the virtue 